\title{
NONWANDERING SETS OF MAPS ON THE CIRCLE
}

\author{
SEUNG WHA YEOM, KYUNG JIN MIN, and SEONG HOON CHO
}

(Received 30 April 1997 and in revised form 25 July 1997)

\begin{abstract}
Let $f$ be a continuous map of the circle $S^{1}$ into itself. And let $R(f), \Lambda(f), \Gamma(f)$, and $\Omega(f)$ denote the set of recurrent points, $\omega$-limit points, $\gamma$-limit points, and nonwandering points of $f$, respectively. In this paper, we show that each point of $\Omega(f) \backslash \overline{R(f)}$ is one-side isolated, and prove that
\end{abstract}

(1) $\Omega(f) \backslash \Gamma(f)$ is countable and

(2) $\Lambda(f) \backslash \Gamma(f)$ and $\overline{R(f)} \backslash \Gamma(f)$ are either empty or countably infinite.

Keywords and phrases. Nonwandering point, recurrent point, one-side isolated.

1991 Mathematics Subject Classification. 58F.

1. Introduction. Let $I$ be the unit interval, $S^{1}$ the circle, and $X$ a topological space. And let $C^{0}(X, X)$ denote the set of continuous maps from $X$ into itself. For any $f \in C^{0}(X, X)$, let $P(f), R(f), \Lambda(f), \Gamma(f)$, and $\Omega(f)$ denote the set of periodic points, recurrent points, $\omega$-limit points, $\gamma$-limit points and nonwandering points of $f$, respectively.

For any $f \in C^{0}(I, I)$, in 1980, Z. Nitecki [6] has proved that each point of $\Omega(f) \backslash \overline{P(f)}$ is isolated in $\Omega(f)$ if $f$ is piecewise monotone and is not flat on any subinterval of $I$. In 1984, J. C. Xiong [7] has proved that each point of $\Omega(f) \backslash \overline{P(f)}$ is one-side isolated in $\Omega(f)$, for a continuous self map of interval $I$. And, in 1988, J. C. Xiong [9] also showed that $\Omega(f) \backslash \Gamma(f)$ is countable and that $\Lambda(f) \backslash \Gamma(f)$ and $\overline{P(f)} \backslash \Gamma(f)$ are either empty or countably infinite.

In this paper, we obtain the following similar results for maps of the circle:

THEOREM 1.1. Let $f \in C^{0}\left(S^{1}, S^{1}\right)$. Then each point of $\Omega(f) \backslash \overline{R(f)}$ is one-side isolated in $\Omega(f)$.

THEOREM 1.2. Let $f \in C^{0}\left(S^{1}, S^{1}\right)$. Then

(1) $\Omega(f) \backslash \Gamma(f)$ is countable.

(2) $\Lambda(f) \backslash \Gamma(f)$ and $\overline{R(f)} \backslash \Gamma(f)$ are either empty or countably infinite.

2. Preliminaries and definitions. Let $X$ be a compact metric space and $f \in C^{0}(X, X)$. For any positive integer $n$, we define $f^{n}$ inductively by $f^{1}=f$ and $f^{n+1}=f \circ f^{n}$. Let $f^{0}$ denote the identity map of $X$. The forward orbit $\operatorname{Orb}(x)$ of $x \in X$ is the set $\left\{f^{k}(x) \mid k=0,1,2, \ldots\right\}$. Usually, the forward orbit of $x$ is simply called the orbit of $x$.

A point $x \in X$ is called a periodic point of $f$ if, for some positive integer $n, f^{n}(x)=x$. The period of $x$ is the least such integer $n$. We denote the set of periodic points of $f$ by $P(f)$. A point $x \in X$ is called a recurrent point of $f$ if there exists a sequence $\left\{n_{i}\right\}$ of positive integers with $n_{i} \rightarrow \infty$ such that $f^{n_{i}}(x) \rightarrow x$. We denote the set of recurrent 
points of $f$ by $R(f)$. A point $x \in X$ is called a nonwandering point of $f$ if, for every neighborhood $U$ of $x$, there exists a positive integer $m$ such that $f^{m}(U) \cap U \neq \phi$. We denote the set of nonwandering points of $f$ by $\Omega(f)$.

A point $y \in X$ is called an $\omega$-limit point of $x$ if there exists a sequence $\left\{n_{i}\right\}$ of positive integers with $n_{i} \rightarrow \infty$ such that $f^{n_{i}}(x) \longrightarrow y$. We denote the set of $\omega$-limit points of $x$ by $\omega(x)$. Define $\Lambda(f)=\bigcup_{x \in X} \omega(x)$. A point $y \in X$ is called an $\alpha$-limit point of $x$ if there exists a sequence $\left\{n_{i}\right\}$ of positive integers with $n_{i} \rightarrow \infty$ and a sequence $\left\{y_{i}\right\}$ of points such that $f^{n_{i}}\left(y_{i}\right)=x$ and $y_{i} \longrightarrow y$. The symbol $\alpha(x)$ denotes the set of $\alpha$-limit points of $x$. A point $y \in X$ is called a $\gamma$-limit point of $x$ if $y \in \omega(x) \cap \alpha(x)$. The symbol $\gamma(x)$ denotes the set of $\gamma$-limit points of $x$ and $\Gamma(f)=\bigcup_{x \in X} \gamma(x)$.

Let $R$ be the set of reals and $Z$ be the set of integers. Formally, we think of the circle $S^{1}$ as $R / Z$ and use $\pi: R \rightarrow R / Z$ to denote the canonical projection. In fact, the map $\pi$ : $R \rightarrow S^{1}$ is an example of a covering map since it wraps $R$ around $S^{1}$ without doubling back (i.e., without critical points). To study the dynamics of the circle map, it is helpful to use a lifting. Let $f$ be a continuous map on the circle. We say that a continuous map $F$ from $R$ into itself is a lifting of $f$ if $f \circ \pi=\pi \circ F$. We use the following notations throughout this paper.

Let $a, b \in S^{1}$ with $a \neq b$, and let $A \in \pi^{-1}(a), B \in \pi^{-1}(b)$ with $|A-B|<1$ and $A<B$. Then we write $\pi((A, B)), \pi([A, B]), \pi([A, B))$ and $\pi((A, B])$ to denote the open, closed, and half-open arcs from $a$ counterclockwise to $b$, respectively, and we denote it by $(a, b),[a, b],[a, b)$, and $(a, b]$. For $x, y \in[a, b]$ with $a \neq b$, let $X \in \pi^{-1}(x), Y \in \pi^{-1}(y)$ with $X, Y \in[A, B]$, then we define for $x, y \in[a, b], x>y$ if and only if $X>Y$. Let $C$ be a subset of a closed arc $[a, b]$, then we define $\sup C=\pi\left(\sup \left(\pi^{-1}(C) \cap[A, B]\right)\right)$ and $\inf C=\pi\left(\inf \left(\pi^{-1}(C) \cap[A, B]\right)\right)$.

In particular, for $a, b, c \in S^{1}, a<b<c$ means that $b$ lies in the open arc $(a, c)$, that is, $b \in(a, c)$.

Let $X$ be $I$ or $S^{1}$ and $Y \subset X$. Let $x \in Y$. A point $x \in X$ is said to be left-sided isolated (resp., right-sided isolated) in $Y$ if, for some $\epsilon>0,(x-\epsilon, x) \cap Y=\phi$ (resp., $(x, x+\epsilon) \cap$ $Y=\phi)$. A point $x$ is said to be one-side isolated in $Y$ if $x$ is either left-side or right-side isolated in $Y$, and a point $x$ which is both a right-sided and a left-sided isolated in $Y$ is said to be isolated in $Y$.

Let $x \in S^{1}$ and $f \in C^{0}\left(S^{1}, S^{1}\right)$ be given. Then we use the symbols $\omega_{+}(x)$ (resp., $\left.\omega_{-}(x)\right)$ to denote the set of all points $y \in S^{1}$ such that there exists a sequence $\left\{n_{i}\right\}$ of positive integers with $n_{i} \rightarrow \infty$ such that $f^{n_{i}}(x) \rightarrow y$ and $y<\cdots<f^{n_{i}}(x)<$ $\cdots<f^{n_{2}}(x)<f^{n_{1}}(x)$ (resp. $f^{n_{1}}(x)<f^{n_{2}}(x)<\cdots<f^{n_{i}}(x)<\cdots<y$ ). It is clear that if $x \notin P(f)$, then $\omega(x)=\omega_{+}(x) \cup \omega_{-}(x)$. Define $\Lambda_{+}(f)=\bigcup_{x \in S^{1}} \omega_{+}(x)$ and $\Lambda_{-}(f)=\bigcup_{x \in S^{1}} \omega_{-}(x)$.

Also, we use the symbols $\alpha_{+}(x)$ (resp. $\alpha_{-}(x)$ ) to denote the set of all points $y \in S^{1}$ such that there exists a sequence $\left\{n_{i}\right\}$ of positive integers with $n_{i} \longrightarrow \infty$ and a sequence $\left\{x_{i}\right\}$ of points such that $x_{i} \longrightarrow y, f^{n_{i}}\left(x_{i}\right)=x$ for every $i>0$ and $y<\cdots<x_{i}<$ $\cdots<x_{2}<x_{1}$ (resp. $x_{1}<x_{2}<\cdots<x_{i}<\cdots<y$ ). It is clear that if $x \notin P(f)$, then $\alpha(x)=\alpha_{+}(x) \cup \alpha_{-}(x)$.

Define $\gamma_{+}(x)=\omega_{+}(x) \cap \alpha_{+}(x)$ and $\gamma_{-}(x)=\omega_{-}(x) \cap \alpha_{-}(x)$. Also, we define $\Gamma_{+}(f)=$ $\bigcup_{x \in S^{1}} \gamma_{+}(x)$ and $\Gamma_{-}(f)=\bigcup_{x \in S^{1}} \gamma_{-}(x)$.

Let $Y$ be an $\operatorname{arc}$ in $S^{1}$ and let $\bar{Y}$ denote the closure of $Y$ as usual. A point $y \in S^{1}$ is 
called a right-sided (resp., left-sided) accumulation point of $Y$ if, for any $z \in S^{1},(y, z) \cap$ $Y \neq \phi$ (resp. $(z, y) \cap Y \neq \phi)$.

The right-side closure $\bar{Y}_{+}$(resp. left-side closure $\bar{Y}_{-}$) is the union of $Y$ and the set of right-sided (resp. left-sided) accumulation points of $Y$. A point which is both a rightsided and a left-sided accumulation point of $Y$ is called a two-sided accumulation point of $Y$.

3. Main results. The following lemmas are founded in [3].

LEMmA 3.1. Let $f \in C^{0}\left(S^{1}, S^{1}\right)$ and $x \in \Omega(f)$. Then we have $x \in \alpha(x)$.

LEMMA 3.2. Let $f \in C^{0}\left(S^{1}, S^{1}\right)$ and $I=[a, b]$ be an arc for some $a, b \in S^{1}$ with $a \neq b$, and let $I \cap P(f)=\phi$.

(a) Suppose that there exists $x \in I$ such that $f(x) \in I$ and $x<f(x)$. Then

(i) if $y \in I, x<y$, and $f(y) \notin[y, b]$, then $[x, y] f$-covers $[f(x), b]$, and

(ii) if $y \in I, y<x$, and $f(y) \notin[y, b]$, then $[y, x] f$-covers $[f(x), b]$.

(b) Suppose that there exists $x \in I$ such that $f(x) \in I$ and $x>f(x)$. Then

(i) if $y \in I, x<y$, and $f(y) \notin[a, y]$, then $[x, y] f$-covers $[a, f(x)]$, and

(ii) if $y \in I, y<x$, and $f(y) \notin[a, y]$, then $[y, x] f$-covers $[a, f(x)]$.

LEMMA 3.3. Let $f \in C^{0}\left(S^{1}, S^{1}\right)$. Then we have

$$
P(f) \subset R(f) \subset \Gamma(f) \subset \overline{R(f)} \subset \Lambda(f) \subset \Omega(f) \subset C R(f) .
$$

The following lemma is due to [5]

LEMMA 3.4. Let $f \in C^{0}\left(S^{1}, S^{1}\right)$, and let $K \subset S^{1}$ with $f(K) \subset K$. If $x \in \Omega(f) \backslash K$, then $f^{n}(x) \notin K^{\circ}$ for any $n \geq 1$.

The idea of the proof of the following lemma is due to [7].

LEMMA 3.5. Let $f \in C^{0}\left(S^{1}, S^{1}\right)$, and let $K \subset S^{1}$ have only finitely many connected components and $f(K)=K$. Then we have $\bar{K} \backslash K \subset P(f)$.

Proof. By continuity of $f$, we have $f(\bar{K}) \subset \overline{f(K)}$. And by the compactness of $\bar{K}, f(\bar{K}) \subset S^{1}$ is closed. Thus, $\overline{f(K)} \subset \overline{f(\bar{K})}=f(\bar{K})$. Therefore, $f(\bar{K})=\overline{f(K)}=\bar{K}$. Hence, for each $x \in \bar{K} \backslash K$, there exists $x^{\prime} \in \bar{K} \backslash K$ such that $f\left(x^{\prime}\right)=x$, i.e., $f(\bar{K} \backslash K)=\bar{K} \backslash K$. By the finiteness of $\bar{K} \backslash K, \bar{K} \backslash K \subset P(f)$.

Proposition 3.6. Let $f \in C^{0}\left(S^{1}, S^{1}\right)$. Suppose that $x \in \Omega(f) \backslash \overline{R(f)}$.

(1) If $x \in \alpha_{+}(x)$, then there exists $z \in S^{1}$ such that $f^{i}(z, x) \cap(z, x)=\phi$ for all $i \geq 1$.

(2) If $x \in \alpha_{-}(x)$, then there exists $u \in S^{1}$ such that $f^{i}(x, u) \cap(x, u)=\phi$ for all $i \geq 1$.

Proof. We only need to prove part (1). There exists $a, b \in S^{1}$ such that $x \in(a, b)$ and $(a, b) \cap \operatorname{Orb}(x)=\phi$. Let $V=(a, x)$ and let $W=\cup_{i=0}^{\infty} f^{i}(V)$. Then $x \in \bar{W}$. Since $x \in$ $\alpha_{+}(x)$, there exist a positive integer $m$ and a point $y \in(x, b)$ such that $f^{m}(y)=x$. By Lemma 3.2,

$$
[x, y] f^{m} \text {-covers }[a, x] .
$$


We claim that $x \notin W$. To show this, suppose that $x \in W$. Then there exist a positive integer $j$ and a point $x_{0} \in(a, x)$ such that $f^{j}\left(x_{0}\right)=x$. By Lemma 3.2,

$$
\left[x_{0}, x\right] f^{j} \text {-covers }[x, b] \text {. }
$$

In particular, $\left[x_{0}, x\right] f^{j}$-covers $[x, y]$.

By (2),

$$
\left[x_{0}, x\right] f^{j} \text {-covers }\left[x_{0}, y\right]
$$

Thus,

$$
\left[x_{0}, x\right] f^{j+m} \text {-covers itself, }
$$

and, hence, $f^{j+m}$ has a periodic point in $(a, b)$, a contradiction. Hence, we have $x \in$ $\bar{W} \backslash W$.

Assume that the proposition is false, i.e., for each $z \in(a, x)$, there is some $i \geq 1$ such that $(z, x) \cap f^{i}(z, x) \neq \phi$. Note that $V \subset f(W)$. Because, for each $y^{\prime} \in V$, there is some $i \geq 1$ such that $\left(y^{\prime}, x\right) \cap f^{i}\left(y^{\prime}, x\right) \neq \phi$. There exists $x_{0} \in\left(y^{\prime}, x\right)$ such that $f^{i}\left(x_{0}\right) \in\left(y^{\prime}, x\right)$. By Lemma 3.2, either

$$
\left[x_{0}, x\right] f^{i} \text {-covers }\left[f^{i}\left(x_{0}\right), b\right] \text { or }\left[x_{0}, x\right] f^{i} \text {-covers }\left[a, f^{i}\left(x_{0}\right)\right] .
$$

Particularly, either

$$
\left[x_{0}, x\right] f^{i} \text {-covers }[x, b] \text { or }\left[x_{0}, x\right] f^{j} \text {-covers }\left[a, f^{i}\left(x_{0}\right)\right] .
$$

If

$$
\left[x_{0}, x\right] f^{i} \text {-covers }[x, b]
$$

then

$$
\left[x_{0}, x\right] f^{j} \text {-covers }[x, y] \text {. }
$$

By (2),

$$
[x, y] f^{m} \text {-covers }\left[x_{0}, x\right] \text {. }
$$

Hence,

$$
\left[x_{0}, x\right] f^{i+m} \text {-covers itself. }
$$

Thus, $f^{j+m}$ has a periodic point in $(a, b)$. This is a contradiction. Therefore,

$$
\left[x_{0}, x\right] f^{j} \text {-covers }\left[a, f^{i}\left(x_{0}\right)\right] \text {. }
$$

Thus, $y^{\prime} \in f^{i}\left(x_{0}, x\right) \subset f^{i}(V) \subset f(W)$ since $y^{\prime} \in\left(a, f^{i}\left(x_{0}\right)\right)$. Thus, for each $i=$ $1,2,3, \ldots, l-1, f^{i}(V) \cap f^{l+i}(V) \neq \phi$, and $f^{l+i}(V) \cap f^{2 l+i}(V) \neq \phi, \ldots$ Therefore, $U_{i}=$ $\cup_{m=0}^{\infty} f^{m l+i}(V)$ is connected and $W=\cup_{i=0}^{l-1} U_{i}$ has only finitely many connected components. Now, by Lemma 3.5, $x \in \bar{W} \backslash W \subset P(f)$. This is in contradiction with the assumption of this proposition. 
The following theorem follows immediately from the proposition.

THEOREM 3.7. Let $f \in C^{0}\left(S^{1}, S^{1}\right)$. Then each point of $\Omega(f) \backslash \overline{R(f)}$ is one-side isolated in $\Omega(f)$.

COROLLARY 3.8. Let $f \in C^{0}\left(S^{1}, S^{1}\right)$. Then $\Omega(f) \backslash \overline{R(f)}$ is countable which is nowhere dense in $S^{1}$.

The following proposition is found in [1].

Proposition 3.9. Let $f \in C^{0}\left(S^{1}, S^{1}\right)$. Then we have

(1) $\overline{R(f)}_{+} \backslash R(f) \subset \Lambda(f)_{+}$.

(2) $\overline{R(f)}_{-} \backslash R(f) \subset \Lambda(f)_{-}$.

Proposition 3.10. Let $f \in C^{0}\left(S^{1}, S^{1}\right)$. Then we have $\overline{R(f)}_{+} \cap \overline{R(f)}_{-} \backslash R(f) \subset \Gamma(f)$.

Proof. If $P(f)=\phi$, then we have the desired results since $\overline{R(f)}=\Gamma(f)$ [2]. Suppose that $P(f) \neq \phi$. Let $z \in \overline{R(f)}_{+} \cap \overline{R(f)}-\backslash R(f)$. Then there exist $a, b \in S^{1}$ with $a<b$ such that $z \in(a, b)$ and $(a, b) \cap \operatorname{Orb}(z)=\phi$. By Proposition 3.9, $z \in \Lambda(f)_{+} \cap \Lambda(f)_{-}$. Then there exist $y_{1}, y_{2}$ such that $a<y_{1}<z<y_{2}<b$ with $z \in \omega\left(y_{1}\right) \cap \omega\left(y_{2}\right)$. Since $\overline{P(f)}=\overline{R(f)}[4], z \in \overline{P(f)}_{+} \cap \overline{P(f)}-\backslash P(f)$. Then there exists $u_{i}$ of periodic point of $f$ with $a<y_{1}<u_{1}<u_{2}<\cdots<z$ and $u_{i} \rightarrow z$. Let $p_{i}$ be the period of $u_{i}$ with respect to $f$. Then $f^{p_{i}}\left(u_{i}\right)=u_{i}$ for all $i \geq 1$. Then either $\left[u_{i}, z\right] f^{p_{i} \text {-covers }\left[a, u_{i}\right] \text { or }}$

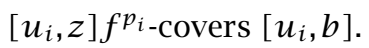

We may assume that, for infinitely many $i$, either

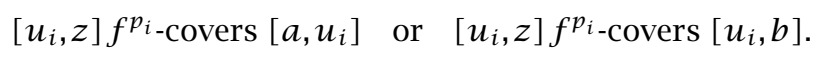

Then we consider two cases.

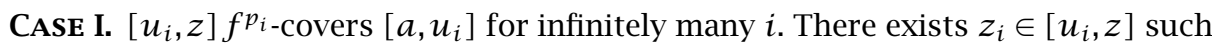
that $f^{p_{i}}\left(z_{i}\right)=y_{1}$. Since $u_{i} \longrightarrow z, z_{i} \rightarrow z$. Thus, $z \in \alpha\left(y_{1}\right)$ and, hence, $z \in \omega\left(y_{1}\right) \cap$ $\alpha\left(y_{1}\right) \subset \Gamma(f)$.

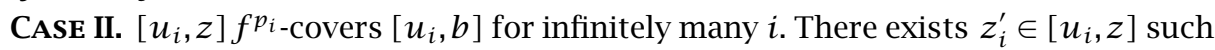
that $f^{p_{i}}\left(z_{i}^{\prime}\right)=y_{1}$. Since $u_{i} \longrightarrow z, z_{i}^{\prime} \rightarrow z$. Thus, $z \in \alpha\left(y_{2}\right)$ and, hence, $z \in \omega\left(y_{2}\right) \cap$ $\alpha\left(y_{2}\right) \subset \Gamma(f)$.

The idea of the proof of the following lemma is due to [8].

LEMMA 3.11. Let $f \in C^{0}\left(S^{1}, S^{1}\right)$ and $Y \subset S^{1}$. Then $\bar{Y} \backslash\left(\bar{Y}+\cap \bar{Y}_{-}\right)$is countable.

Proof. For each $y \in \bar{Y}_{+} \backslash \bar{Y}_{-}$, there is some $u_{y} \in S^{1}$ such that $\left(u_{y}, y\right) \cap Y=\phi$. The family of $\left\{\left(u_{y}, y\right) \mid y \in \bar{Y}_{+} \backslash \bar{Y}_{-}\right\}$is countable because it is disjoint. Hence, $\bar{Y}_{+} \backslash \bar{Y}_{-}$is countable. Similarly, $\bar{Y}_{-} \backslash \bar{Y}_{+}$is also countable. Therefore,

$$
\bar{Y} \backslash\left(\bar{Y}_{+} \cap \bar{Y}_{-}\right)=\left(\bar{Y}_{+} \backslash \bar{Y}_{-}\right) \cup\left(\bar{Y}_{-} \backslash \bar{Y}_{+}\right)
$$

is countable.

THEOREM 3.12. Let $f \in C^{0}\left(S^{1}, S^{1}\right)$. Then

(1) $\Omega(f) \backslash \Gamma(f)$ is countable.

(2) $\Lambda(f) \backslash \Gamma(f)$ and $\overline{R(f)} \backslash \Gamma(f)$ are either empty or countably infinite. 
Proof. (1) We know that $\overline{R(f)} \backslash\left(\overline{R(f)_{+}} \cap \overline{R(f)_{-}}\right)$is countable by Lemma 3.11 . By Proposition 3.10, $\overline{R(f)} \backslash \Gamma(f)$ is also countable. By Corollary 3.8, $\Omega(f) \backslash \overline{R(f)}$ is countable. Hence, $\Omega(f) \backslash \Gamma(f)$ is countable.

(2) It is easy to prove that $f(\omega(x))=\omega(x)$ and $f(\overline{R(f)})=\overline{R(f)}$ for $x \in S^{1}$. Hence, $f(\Lambda(f))=\Lambda(f)$. Suppose that $\Lambda(f) \backslash \Gamma(f) \neq \phi$ (resp., $\overline{R(f)} \backslash \Gamma(f) \neq \phi)$. Then we take $z_{1} \in \Lambda(f) \backslash \Gamma(f)$ (resp., $z_{1} \in \overline{R(f)} \backslash \Gamma(f)$ ). We can take $z_{2} \in \Lambda(f) \backslash \Gamma(f)$ (resp., $\left.z_{2} \in \overline{R(f)} \backslash \Gamma(f)\right)$ such that $z_{1}=f\left(z_{2}\right)$. Continuing this process, we can take $z_{i} \in$ $\Lambda(f) \backslash \Gamma(f)$ (resp., $\left.z_{i} \in \overline{R(f)} \backslash \Gamma(f)\right)$ such that $z_{i}=f\left(z_{i+1}\right)$ for all $i=1,2, \ldots$. Since $z_{i} \notin(f)$ for all $i \geq 1$, the points $z_{1}, z_{2}, \ldots$ are pairwise disjoint. Hence, $\Lambda(f) \backslash \Gamma(f)$ (resp., $\overline{R(f)} \backslash \Gamma(f)$ ) is infinite and, hence, $\Lambda(f) \backslash \Gamma(f)$ (resp., $\overline{R(f)} \backslash \Gamma(f)$ ) is countably infinite.

ACKNOWLEDGEMENT. This research was supported by fund of SNUT.

\section{REFERENCES}

[1] J. S. Bae, S. H. Cho, K. J. Min, and S. K. Yang, Nonwandering points and the attracting centre of a map on the circle, to appear.

[2] _ Nonwandering points of a map on the circle, J. Korean Math. Soc. 33 (1996), no. 4, 1115-1121. MR 97m:58061. Zbl 871.58031.

[3] J. S. Bae, S. H. Cho, and S. K. Yang, Relations between nonwandering points and turning points on the circle, Bull. Korean Math. Soc. 32 (1995), no. 1, 57-66. MR 96b:58028. Zbl 836.54026.

[4] J. S. Bae and S. K. Yang, $\bar{P}=\bar{R}$ for maps of the circle, Bull. Korean Math. Soc. 24 (1987), 151-157. Zbl 718.54047.

[5] _,$\omega$-limit sets for maps of the circle, Bull. Korean Math. Soc. 25 (1988), no. 2, 233-242. MR 90m:58164. Zbl 681.54020.

[6] Z. Nitecki, Periodic and limit orbits and the depth of the center for piecewise monotone interval maps, Proc. Amer. Math. Soc. 80 (1980), no. 3, 511-514. MR 81j:58068. Zbl 478.58019 .

[7] J. C. Xiong, Nonwandering sets of continuous interval self-maps, Kexue Tongbao (English Ed.) 29 (1984), no. 11, 1431-1433. MR 86j:58131. Zbl 575.54034.

[8] _ Sets of recurrent points of continuous maps of the interval, Proc. Amer. Math. Soc. 95 (1985), no. 3, 491-494. MR 87d:58114. Zbl 591.54031.

[9] _ The attracting centre of a continuous self-map of the interval, Ergodic Theory Dynamical Systems 8 (1988), no. 2, 205-213. MR 90b:58131. Zbl 658.58032.

Yeom and Min: Department of Mathematics, Seoul National University of TeChNOLOGY, NOWON-Gu, SEOUL, 139-743, KOREA

Cho: Department of Mathematics, Hanseo University, Chungnam, Seosan, 356-820, KOREA 


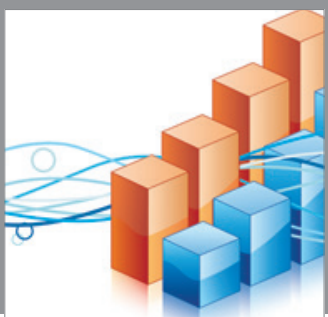

Advances in

Operations Research

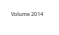

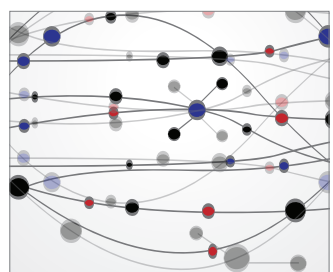

\section{The Scientific} World Journal
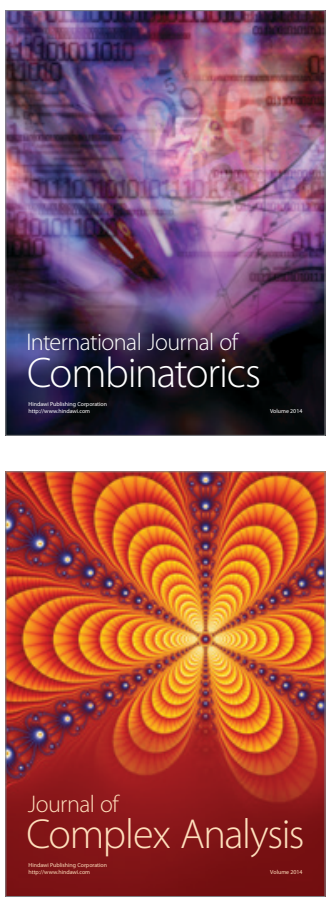

International Journal of

Mathematics and

Mathematical

Sciences
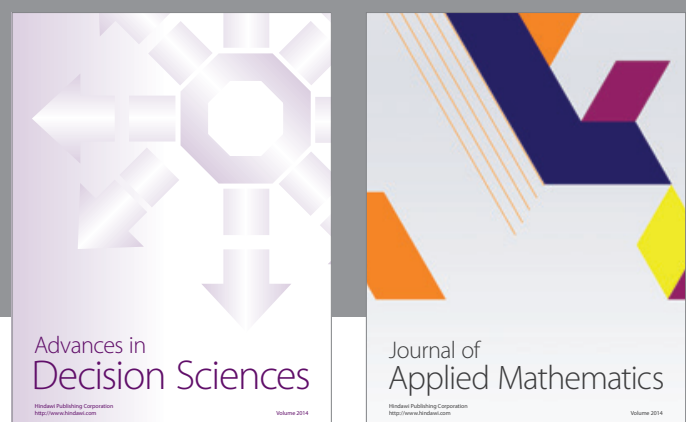

Journal of

Applied Mathematics
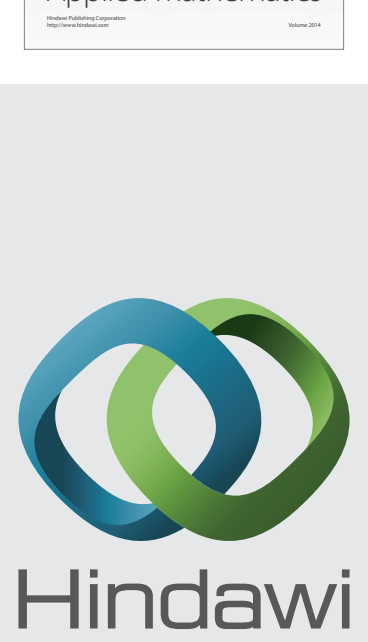

Submit your manuscripts at http://www.hindawi.com
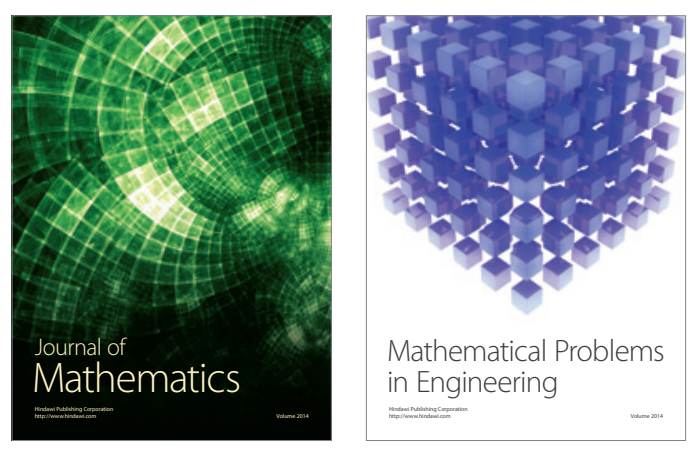

Mathematical Problems in Engineering
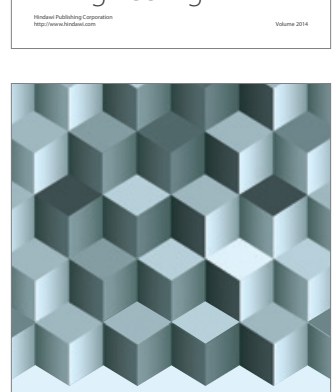

Journal of

Function Spaces
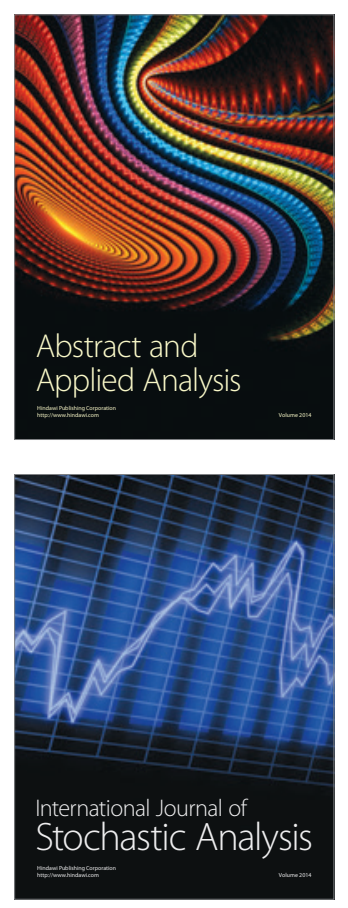

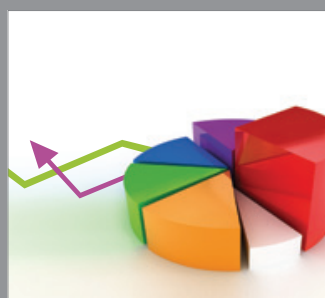

ournal of

Probability and Statistics

Promensencen
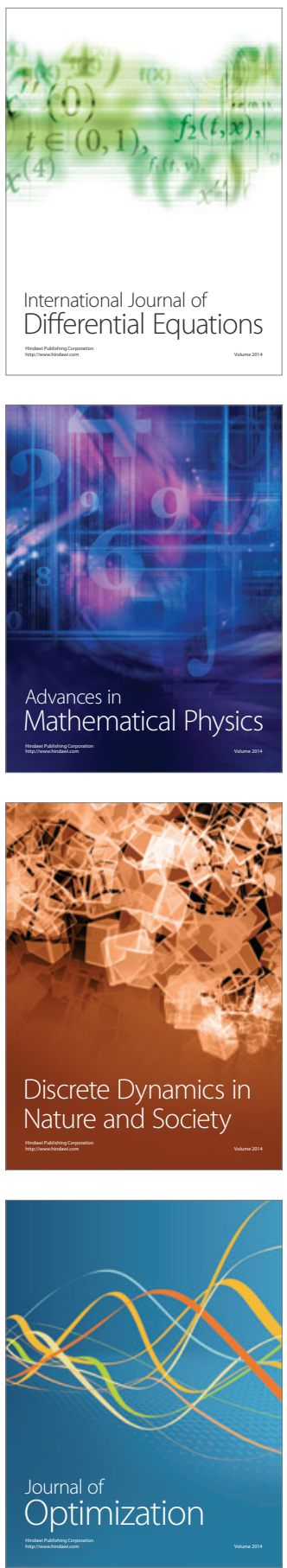